\title{
Segurança e Factibilidade do Acesso Ulnar Após Falha do Acesso Radial Ipsilateral
}

\author{
Pedro Beraldo de Andrade ${ }^{1}$, Fábio Salerno Rinaldi ${ }^{1}$, Igor Ribeiro de Castro Bienert ${ }^{2}$, \\ Ederlon Ferreira Nogueira ${ }^{3}$, Marden André Tebet ${ }^{4}$, Vinícius Cardozo Esteves ${ }^{4}$, \\ Mônica Vieira Athanazio de Andrade', Robson Alves Barbosa', Luiz Alberto Piva e Mattos ${ }^{4}$, André Labrunie ${ }^{3}$
}

\section{RESUMO}

Introdução: Publicações pioneiras desencorajam o uso do acesso ulnar após falha na tentativa de obtenção do acesso radial ipsilateral. Métodos: Registro prospectivo de eficácia e segurança comparando a ocorrência de sangramento e complicações vasculares entre casos com intenção inicial de utilizar o acesso ulnar e aqueles cujo emprego desse acesso se deu após falha na canulação da artéria radial ipsilateral. Resultados: Entre maio de 2007 e dezembro de 2013, foram realizados 11.825 procedimentos coronários invasivos em um único centro, 473 (4\%) deles pelo acesso ulnar. Em 65 casos, a canulação da artéria ulnar foi precedida por falha na obtenção do acesso radial ipsilateral. A taxa de sucesso da técnica foi superior a $98 \%$, sendo baixo o número de complicações, em sua maioria decorrentes de hematomas superficiais, sem diferença entre os grupos. Não houve casos de sangramento grave relacionado a via de acesso, pseudoaneurisma, fístula arteriovenosa ou lesão do nervo ulnar adjacente. Conclusões: $\mathrm{O}$ acesso ulnar representa uma alternativa segura e eficaz para a realização de procedimentos coronários invasivos selecionados, a despeito de tentativa prévia de obtenção do acesso radial ipsilateral.

DESCRITORES: Artéria ulnar. Artéria radial. Angiografia coronária. Intervenção coronária percutânea. Hemorragia.

A escolha da via de acesso para a realização de procedimentos coronários invasivos configura uma importante etapa dentro de uma abordagem que contemple a redução de complicações vasculares e de sangramento grave. Embora a técnica femoral se mantenha preponderante, observa-se um crescimento consistente na adoção da técnica radial, sabidamente promotora de maior conforto e segurança ao paciente. ${ }^{1,2}$
ABSTRACT

\section{Safety and Feasibility of Ulnar Access After Failure of Ipsilateral Radial Access}

Background: Pioneering publications discourage the use of ulnar access after failed attempt to obtain ipsilateral radial access. Methods: Prospective efficacy and safety registry comparing the incidence of bleeding and vascular complications in patients with an initial intention to use ulnar access and those who used this access after a failed attempt to cannulate the ipsilateral radial artery. Results: Between May 2007 and December 2013, 11,825 invasive coronary procedures were performed at a single center, $473(4 \%)$ of them by ulnar access. In 65 cases, the ulnar artery cannulation was preceded by a failed attempt to obtain the ipsilateral radial access. The technical success rate was higher than $98 \%$, with low complication rates, mostly due to superficial hematomas, and no differences between groups. There were no cases of major bleeding related to the access site, pseudoaneurysm, arteriovenous fistula or injury to the adjacent ulnar nerve. Conclusions: The ulnar access is a safe and effective alternative to perform selected invasive coronary procedures, despite previous attempts to obtain the ipsilateral radial access.

DESCRIPTORS: Ulnar artery. Radial artery. Coronary angiography. Percutaneous coronary intervention. Hemorrhage.

Espasmo, variações anatômicas e menor amplitude de pulso, consequentes à reutilização da via, constituem mecanismos de insucesso na obtenção do acesso radial. ${ }^{3}$ Nesse contexto, a artéria ulnar representa uma alternativa viável capaz de ofertar benefícios semelhantes àqueles advindos da técnica radial, conforme demonstrado em registros prévios. ${ }^{4,5}$ Entretanto, publicações pioneiras acerca do tema desencorajam o uso do acesso ulnar após falha na canulação da artéria radial ipsilateral. ${ }^{6,7}$

\footnotetext{
' Irmandade da Santa Casa de Misericórdia de Marília, Marília, SP, Brasil.

2 Hospital das Clínicas, Faculdade de Medicina de Marília, Marília, SP, Brasil.

${ }^{3}$ Hospital do Coração de Londrina, Londrina, PR, Brasil.

${ }^{4}$ Rede D’Or São Luiz, São Paulo, SP, Brasil.
}

\author{
Correspondência: Pedro Beraldo de Andrade. Avenida Vicente Ferreira \\ 828 - CEP 17515-900 - Marília, SP, Brasil \\ E-mail: pedroberaldo@gmail.com \\ Recebido em: 19/12/2013 • Aceito em: 25/2/2014
}


O objetivo desta análise foi avaliar a factibilidade e a segurança da realização de procedimentos coronários pela artéria ulnar em situações de falha na tentativa de utilizar a artéria radial ipsilateral.

\section{MÉTODOS}

Pacientes consecutivos encaminhados para a realização de procedimentos coronários invasivos que utilizaram a via ulnar foram incluídos em um registro prospectivo de segurança e eficácia. O objetivo primário do estudo foi a comparação de características clínicas, características relacionadas ao procedimento, e ocorrência de sangramento e de complicações vasculares entre os casos com intenção inicial de punção da artéria ulnar (Grupo 1) e aqueles cujo emprego se deu após falha na obtenção do acesso radial ipsilateral (Grupo 2).

\section{Definições}

Em conformidade com a classificação do Bleeding Academic Research Consortium (BARC), ${ }^{8}$ sangramento grave foi definido como do tipo 3 - (3a) sangramento com queda de hemoglobina $\geq 3$ e $<5 \mathrm{~g} / \mathrm{dL}$, ou transfusão de concentrado de hemácias; (3b) sangramento com queda de hemoglobina $\geq 5 \mathrm{~g} / \mathrm{dL}$, ou tamponamento cardíaco, ou sangramento que requeira intervenção cirúrgica, ou sangramento que requeira uso de drogas vasoativas intravenosas; (3c) hemorragia intracraniana, ou subcategorias confirmadas por autópsia, exame de imagem, ou punção lombar, ou sangramento intraocular com comprometimento da visão - ou do tipo 5 - (5a) sangramento fatal provável; (5b) sangramento fatal definitivo.

Os hematomas foram graduados de acordo com a classificação do estudo Early Discharge After Transradial Stenting of Coronary Arteries (EASY): tipo I, $\leq 5 \mathrm{~cm}$ de diâmetro; tipo II, $\leq 10 \mathrm{~cm}$ de diâmetro; tipo III, $>10 \mathrm{~cm}$, sem atingir o cotovelo; tipo IV, hematoma estendendo-se além do cotovelo; tipo $V$, qualquer hematoma com injúria isquêmica à mão. ${ }^{9}$ Complicações relacionadas ao sítio de punção, além de hematomas, incluíram oclusão arterial assintomática, lesão do nervo ulnar, isquemia aguda, pseudoaneurisma, fístula arteriovenosa, necessidade de reparo vascular cirúrgico e infecção local.

O sucesso da técnica foi definido como conclusão do procedimento diagnóstico ou terapêutico sem necessidade de cruzamento para outra via de acesso. Oclusão arterial assintomática foi definida como a interrupção do fluxo sanguíneo arterial, sem manifestações de comprometimento isquêmico da extremidade. Lesão do nervo ulnar foi definida como a ocorrência de distúrbios sensitivos e/ou motores no membro no qual o procedimento foi realizado, com persistência dos sinais e/ou sintomas por período $\geq 24$ horas, como consequência de lesão direta por punção inadvertida do nervo ou compressão extrínseca por hematoma e/ou pseudoaneurisma. A duração do procedimento e o tem- po de fluoroscopia foram obtidos a partir da punção arterial até a retirada do introdutor.

\section{Técnica ulnar}

Por meio da hiperextensão do punho e infiltração de 1 a $2 \mathrm{~mL}$ de xilocaína a $2 \%$, puncionou-se a artéria ulnar 1 a $3 \mathrm{~cm}$ proximal ao osso pisiforme, utilizando-se agulha com cateter de polietileno tamanho 20-22 e técnica de Seldinger. Após a punção, um fio-guia de 0,019 polegada foi introduzido, seguido de pequena incisão cutânea com lâmina de bisturi número 11 e inserção de introdutor curto 5 ou 6 F. Solução contendo $5.000 \mathrm{UI}$ de sulfato de heparina e $10 \mathrm{mg}$ de mononitrato de isossorbida foi injetada por meio da extensão do introdutor. Ao término do procedimento, o introdutor foi imediatamente removido e a hemostasia foi obtida com curativo compressivo com bandagem elástica adesiva porosa nos procedimentos diagnósticos e pulseira compressora seletiva adaptada nos procedimentos terapêuticos. Foram realizados o exame clínico do sítio de punção e a avaliação do pulso ulnar no momento da alta, reservando o uso do ultrassom Doppler para suspeita de complicações.

\section{Análise estatística}

Variáveis qualitativas foram resumidas em frequências absolutas e porcentagens, e as variáveis quantitativas foram descritas como médias \pm desvios padrão. Para comparação dos grupos, foi utilizado o teste qui-quadrado ou exato de Fisher para as variáveis qualitativas; o teste $t$ de Student foi usado para as variáveis quantitativas. Valores de $p<0,05$ foram considerados significantes.

\section{RESULTADOS}

Entre maio de 2007 e dezembro de 2013, foram realizados 11.825 procedimentos coronários invasivos em um único centro, dos quais 10.761 (91\%) pelo acesso radial, 591 (5\%) pelo acesso femoral e 473 (4\%) pelo acesso ulnar - os últimos representando a amostra analisada.

Nos dois grupos, houve preponderância de pacientes do sexo feminino, com média de idades de $61 \pm 11,2$ anos, e elevada prevalência de diabéticos. De maneira geral, os grupos mostraram características idênticas, com exceção de hipertensão arterial e doença vascular prévia, menos frequentes nos pacientes com cruzamento da via radial para a ulnar. Esses pacientes também exibiram menor porcentual de intervenções coronárias percutâneas primárias (Tabela 1).

Procedimentos diagnósticos responderam por $73 \%$ dos casos, e a via ulnar direita foi utilizada em quatro de cada cinco pacientes, mas foi menos frequentemente nos casos de cruzamento da via radial para a via ulnar $(88,7 \%$ vs. $78,5 \% ; p=0,02)$. O número de cateteres utilizados nos procedimentos foi semelhante e o introdutor $5 \mathrm{~F}$ foi utilizado em pelo menos dois terços das intervenções. Os tempos do procedimento e fluoroscopia não diferiram entre os grupos (Tabela 2). 
A taxa de sucesso da técnica foi superior a $98 \%$, sendo baixo o número de complicações, em sua maioria decorrentes de hematomas superficiais, sem injúria isquêmica à mão. Não houve casos de sangramento grave relacionado à via de acesso, pseudoaneurisma, fístula arteriovenosa e nem lesão do nervo ulnar adjacente (Tabela 3).

\section{DISCUSSÃO}

Relatos pioneiros acerca da utilização da artéria ulnar como nova opção de acesso vascular em procedimentos invasivos caracterizam-se pelo pequeno número de pacientes e por advertências quanto a sua escolha em situações de insucesso na obtenção do acesso radial ipsilateral, canulação prévia do mesmo no passado e avaliação mandatória da permeabilidade do arco palmar por meio do teste de Allen ou pletismografia. ${ }^{6,10,11}$ Com a maior experiência obtida sobretudo por grupos que privilegiam e adotam a técnica radial como primeira escolha, e que, consequentemente, migraram para a técnica ulnar diante da necessidade de uma via alternativa, comprovaram-se sua segurança e sua eficácia, mesmo em situações limítrofes. ${ }^{12-14}$

Registro prospectivo recente avaliou 240 pacientes submetidos a procedimentos vasculares diagnósticos e terapêuticos pelo acesso ulnar a despeito de documentação prévia de oclusão do acesso radial ipsilateral. ${ }^{15}$ Os autores reportaram taxa de sucesso de 97\%, com necessidade de cruzamento para o acesso femoral em $3 \%$ dos casos. Apesar da ocorrência de espasmo em $8 \%$ dos pacientes e taxa de oclusão assintomática da artéria ulnar de $3 \%$, não houve relato de sinais de

TABELA 1

Características clínicas basais

\begin{tabular}{|c|c|c|c|c|}
\hline Variáveis & $\begin{array}{c}\text { Geral } \\
(n=473)\end{array}$ & $\begin{array}{c}\text { Grupo } 1 \\
(n=408)\end{array}$ & $\begin{array}{l}\text { Grupo } 2 \\
(n=65)\end{array}$ & Valor de $p$ \\
\hline Sexo masculino, $n(\%)$ & $220(46,5)$ & $189(46,3)$ & $31(47,7)$ & 0,89 \\
\hline Idade, anos & $61,6 \pm 11,2$ & $61,9 \pm 11,3$ & $59,6 \pm 10,8$ & 0,12 \\
\hline Hipertensão arterial sistêmica, n (\%) & $397(83,9)$ & $349(85,5)$ & $48(73,8)$ & 0,02 \\
\hline Diabetes mellitus, $\mathrm{n}(\%)$ & $173(36,6)$ & $154(37,7)$ & $19(29,2)$ & 0,21 \\
\hline Dislipidemia, n (\%) & $277(58,6)$ & $240(58,8)$ & $37(56,9)$ & 0,78 \\
\hline Tabagismo atual, n (\%) & $115(24,3)$ & $97(23,8)$ & $18(27,7)$ & 0,53 \\
\hline Doença vascular prévia*, n (\%) & $154(32,6)$ & $141(34,6)$ & $13(20,0)$ & 0,02 \\
\hline Quadro clínico, n (\%) & & & & $<0,01$ \\
\hline Angina estável & $260(55,0)$ & $224(54,9)$ & $36(55,4)$ & \\
\hline SCASST & $153(32,3)$ & $125(30,6)$ & $28(43,1)$ & \\
\hline IAMCST & $60(12,7)$ & $59(14,5)$ & $1(1,5)$ & \\
\hline
\end{tabular}

* Infarto agudo do miocárdio, acidente vascular encefálico, intervenção coronária percutânea ou revascularização miocárdica cirúrgica prévias.

SCASST: síndrome coronária aguda sem supradesnivelamento do segmento ST; IAMCST: infarto agudo do miocárdio com supradesnivelamento do segmento ST.

TABELA 2

Características dos procedimentos

\begin{tabular}{|c|c|c|c|c|}
\hline Variáveis & $\begin{array}{c}\text { Geral } \\
(n=473)\end{array}$ & $\begin{array}{l}\text { Grupo } 1 \\
(n=408)\end{array}$ & $\begin{array}{r}\text { Grupo } 2 \\
(n=65)\end{array}$ & Valor de $p$ \\
\hline Tipos, n (\%) & & & & 0,36 \\
\hline Diagnóstico & $345(72,9)$ & $294(72,1)$ & $51(78,5)$ & \\
\hline Terapêutico & $128(27,1)$ & $114(27,9)$ & $14(21,5)$ & \\
\hline Ulnar direita, n (\%) & $413(87,3)$ & $362(88,7)$ & $51(78,5)$ & 0,02 \\
\hline Número de cateteres & $1,9 \pm 0,8$ & $1,9 \pm 0,9$ & $2,0 \pm 0,6$ & 0,38 \\
\hline Calibre do introdutor, $\mathrm{n}(\%)$ & & & & 0,38 \\
\hline $5 \mathrm{~F}$ & $324(68,5)$ & $276(67,6)$ & $48(73,8)$ & \\
\hline $6 \mathrm{~F}$ & $149(31,5)$ & $132(32,4)$ & $17(26,2)$ & \\
\hline Duração, minutos & $24,8 \pm 15,1$ & $24,5 \pm 15,6$ & $26,3 \pm 11,7$ & 0,37 \\
\hline Tempo de fluoroscopia, minutos & $5,3 \pm 5,2$ & $5,4 \pm 5,4$ & $4,4 \pm 3,2$ & 0,14 \\
\hline
\end{tabular}


TABELA 3

Comparações entre procedimentos realizados pelo acesso ulnar como opção inicial e após insucesso do acesso radial ipsilateral (desfechos de eficácia/segurança)

\begin{tabular}{|c|c|c|c|c|}
\hline Variáveis & $\begin{array}{c}\text { Geral } \\
(n=473)\end{array}$ & $\begin{array}{c}\text { Grupo } 1 \\
(n=408)\end{array}$ & $\begin{array}{c}\text { Grupo } 2 \\
(n=65)\end{array}$ & Valor de $p$ \\
\hline Sucesso da técnica, n (\%) & $467(98,7)$ & $403(98,8)$ & $64(98,5)$ & 0,59 \\
\hline Tipo de hematoma, n (\%) & $15(3,2)$ & $12(2,9)$ & $3(4,6)$ & 0,44 \\
\hline 1 & $10(66,7)$ & $8(66,7)$ & $2(66,7)$ & \\
\hline ॥ & $1(6,7)$ & $1(8,3)$ & 0 & \\
\hline III & $2(13,3)$ & $1(8,3)$ & $1(33,3)$ & \\
\hline IV & $2(13,3)$ & $2(16,7)$ & 0 & \\
\hline V & 0 & 0 & 0 & \\
\hline Oclusão arterial assintomática, n (\%) & $4(0,8)$ & $4(0,9)$ & 0 & $>0,99$ \\
\hline Outras complicações vasculares ${ }^{\star}, \mathrm{n}(\%)$ & 0 & 0 & 0 & NA \\
\hline
\end{tabular}

* Sangramento grave, pseudoaneurisma, fístula arteriovenosa, lesão do nervo ulnar. NA: não aplicável.

isquemia da mão aos 30 dias de seguimento. De fato, a presença do arco palmar profundo em mais de $95 \%$ dos estudos anatômicos e a capacidade de reserva vascular da circulação da mão, demonstrada pelo recrutamento de colaterais em modelos de laboratório, justificariam a ausência de complicações isquêmicas pós-procedimento. ${ }^{16}$

Na presente casuística, cujos resultados principais se encontram previamente publicados, ${ }^{4}$ demonstramos que, a despeito do espasmo da artéria radial em $80 \%$ dos casos de insucesso e do posterior cruzamento para a artéria ulnar ipsilateral, essa via foi utilizada sem implicações negativas para o paciente, sobretudo quanto ao risco de injúria isquêmica à mão. A possibilidade de efetivar o procedimento mais rapidamente em situações emergenciais, como na intervenção coronária percutânea primária, na qual a necessidade de preparo do membro superior contralateral ou do membro inferior acarretaria atraso adicional para início do procedimento, a utilização dos mesmos materiais previamente empregados na tentativa de punção da artéria radial e a maior comodidade para o paciente e o operador justificariam a opção pela via ulnar.

Resultados similares foram relatados em uma série de 12 pacientes submetidos à angiografia sistemática do arco palmar ao término de procedimentos invasivos pelo acesso ulnar ipsilateral, após falha do acesso radial. ${ }^{13} \mathrm{Em}$ sete casos, a artéria radial estava patente, indicando um espasmo temporário. Um paciente apresentava oclusão crônica da artéria radial, com ramos colaterais provenientes de ambas as artérias. Nos quatro casos restantes, constatou-se oclusão radial aguda por possível dissecção, mas com clara demonstração de circulação colateral para o arco palmar, sem evidência clínica de isquemia.

Em nossa prática diária, na impossibilidade de utilização do acesso radial, advogamos o uso do acesso ulnar, independentemente da constatação de oclusão ou da falha de canulação da artéria radial ipsilateral, sendo o único requisito a presença de um pulso amplo e facilmente identificável, o que representa menos de 5\% do total de nossos procedimentos. A opção pela técnica ulnar sem avaliação prévia da anatomia e do pulso resultará em maior número de tentativas de punção, tempo para obtenção do acesso, duração do exame e taxa de insucesso próxima a 45\%. ${ }^{17,18}$

Dentre as limitações do nosso estudo, ressaltamos sua natureza não randômica, o tamanho amostral e, sobretudo, tratar-se de uma casuística de um único centro, caracterizado por operadores familiarizados com o acesso radial e ulnar, utilizados em 95\% dos procedimentos, bem como a disponibilidade de materiais dedicados a essas vias, como introdutores e cateteres hidrofílicos, e equipe multidisciplinar experiente e treinada no manuseio de sua hemostasia.

\section{CONCLUSÕES}

O acesso ulnar representou uma alternativa segura e eficaz para a realização de procedimentos coronários invasivos em casos selecionados, mesmo após falha na tentativa de obtenção do acesso radial ipsilateral.

\section{CONFLITO DE INTERESSES}

Não há.

\section{FONTE DE FINANCIAMENTO}

Não há.

\section{REFERÊNCIAS}

1. Andrade PB, Tebet MA, Andrade MVA, Labrunie A, Mattos LAP. Acesso radial em intervenções coronarianas percutâneas: panorama atual brasileiro. Arq Bras Cardiol. 2011;96(4):312-6. 
2. Rinaldi FS, Andrade PB, Andrade MVA, Mattos LAP, Santucci $E V$, Cavalcante MA, et al. Farmacoterapia antitrombótica intra-hospitalar e aos seis meses após intervenção coronária percutânea primária: análise do registro da prática clínica em síndrome coronária aguda (ACCEPT). Rev Bras Cardiol Invasiva. $2013 ; 21(1): 30-5$

3. Dehghani P, Mohammad A, Bajaj R, Hong T, Suen CM, Sharieffet $W$, et al. Mechanism and predictors of failed transradial approach for percutaneous coronary interventions. JACC Cardiovasc Interv. 2009;2(11):1057-64.

4. Andrade PB, Tebet MA, Nogueira EF, Esteves VC, Andrade MVA, Labrunie A, et al. Transulnar approach as an alternative access site for coronary invasive procedures after transradial approach failure. Am Heart J. 2012;164(4):462-7.

5. Andrade PB, Tebet MA, Andrade MVA, Mattos LAP, Labrunie A. Registro prospectivo de avaliação da segurança e eficácia da técnica ulnar na realização de procedimentos coronários. Rev Bras Cardiol Invasiva. 2008;16(3):312-6.

6. Dashkoff N, Dashkoff PB, Zizzi JA Sr, Wadhwani J, Zizzi JA Jr. Ulnar artery cannulation for coronary angiography and percutaneous coronary intervention: case reports and anatomic considerations. Cathet Cardiovasc Interv. 2002;55(1):93-6.

7. Aptecar E, Dupouy P, Chabane-Chaouch M, Bussy N, Catarino G, Shahmir A, et al. Percutaneous transulnar artery approach for diagnostic and therapeutic coronary intervention. J Invasive Cardiol. 2005;17(6):312-7.

8. Mehran R, Rao SV, Bhatt DL, Gibson CM, Caixeta A, Eikelboom J, et al. Standardized bleeding definitions for cardiovascular clinical trials: a consensus report from the Bleeding Academic Research Consortium. Circulation. 2011;123(23):2736-47.

9. Bertrand OF, De Larochellière R, Cabau JR, Proulx G, Gleeton $\mathrm{O}$, Nguyen $\mathrm{CM}$, et al. A randomized study comparing same-day home discharge and abciximab bolus only to overnight hospitalization and abciximab bolus and infusion after transradial coronary stent implantation. Circulation. 2006;114(24):2636-43.
10. Terashima $M$, Meguro $T$, Takeda $H$, Endoh N, Ito $\mathrm{Y}$, Mitsuoka $M$, et al. Percutaneous ulnar artery approach for coronary angiography: a preliminary report in nine patients. Cathet Cardiovasc Interv. 2001;53(3):410-4.

11. Limbruno U, Rossini R, De Carlo M, Amoroso G, Ciabatti $\mathrm{N}$, Petronio AS, et al. Percutaneous ulnar artery approach for primary coronary angioplasty: safety and feasibility. Catheter Cardiovasc Interv. 2004;61(1):56-9.

12. Agostoni P, Zuffi A, Biondi-Zoccai G. Pushing wrist access to the limit: homolateral right ulnar artery approach for primary percutaneous coronary intervention after right radial failure due to radial loop. Catheter Cardiovasc Interv. 2011;78(6):894-7.

13. Lanspa TJ, Willians MA, Heirigs RL. Effectiveness of ulnar artery catheterization after failed attempt to cannulate a radial artery. Am J Cardiol. 2005;95(12):1529-30.

14. Andrade PB, Tebet MA, Andrade MV, Labrunie A, Mattos LA. Primary percutaneous coronary intervention through transulnar approach: safety and effectiveness. Arq Bras Cardiol. 2008;91(4):e41-4,e49-52.

15. Kedev S, Zafirovska B, Dharma S, Petkoska D. Safety and feasibility of transulnar catheterization when ipsilateral radial access is not available. Catheter Cardiovasc Interv. 2014; 83(1):E51-60

16. Andrade PB, Tebet MA, Andrade MVA, Mattos LAP, Labrunie A. Performance of coronary procedures through the transulnar access without assessment of the integrity of the deep palmar arch. J Interv Cardiol. 2008;21(6):562-5.

17. Vassilev D, Smilkova D, Gil R. Ulnar artery as access for cardiac catheterization: anatomical considerations. J Interv Cardiol. 2008;21(1):56-60.

18. Hahalis G, Tsigkas G, Xanthopoulou I, Deftereos S, Ziakas A, Raisakis K, et al. Transulnar compared with transradial artery approach as a default strategy for coronary procedures: A randomized trial. The Transulnar or Transradial Instead of Coronary Transfemoral Angiographies Study (The AURA of ARTEMIS Study). Circ Cardiovasc Interv. 2013;6(3):252-61. 